\title{
A NOTE ON THE UNIQUENESS RESULT FOR THE INVERSE HENDERSON PROBLEM*
}

\author{
FABIO FROMMER ${ }^{\dagger}$, MARTIN HANKE $^{\ddagger}$, AND SABINE JANSEN ${ }^{\S}$
}

\begin{abstract}
The inverse Henderson problem of statistical mechanics concerns classical particles in continuous space which interact according to a pair potential depending on the distance of the particles. Roughly stated, it asks for the interaction potential given the equilibrium pair correlation function of the system. In 1974 Henderson proved that this potential is uniquely determined in a canonical ensemble and he claimed the same result for the thermodynamical limit of the physical system. Here we provide a rigorous proof of a slightly more general version of the latter statement using Georgii's version of the Gibbs variational principle.
\end{abstract}

Key words. Statistical mechanics, Gibbs variational principle, radial distribution function

AMS subject classifications. $82 \mathrm{~B} 21$

1. Introduction. An important inverse problem in computational physics and computational chemistry is the determination of the interacting forces in a system of particles in continuous space, given structural information on the spatial distribution of the particles. In the simplest incarnation of this problem it is assumed that the potential energy of the particle ensemble is determined by a pair potential which only depends on the distance of the interacting particles. In an often cited paper Henderson [10] has claimed that under given conditions of temperature and density this pair potential is uniquely determined by the so-called radial distribution function, which - suitably normalized - assigns to each $r>0$ the expected number of particles on a sphere of radius $r$ around any given particle. Roughly speaking, the radial distribution function is obtained from the pair correlation function (called pair density function in the physical literature) associated with a canonical or grand canonical ensemble in a finite volume, when taking the limit of the volume to infinity, the socalled thermodynamical limit. To give credit to Henderson's contribution, this inverse problem of statistical mechanics is sometimes called the inverse Henderson problem.

Henderson's argument makes use of a technique suggested by Hohenberg and Kohn [11], Mermin [16], and others, for studying a similar inverse problem for external potentials. The key idea is to apply a Gibbs variational principle, which states that in a system with given thermodynamic conditions the associated thermodynamic potential becomes minimal, if and only if the distribution of the particles is given by the probability measure associated with this system. The particular version of this principle used by Henderson is based on the free energy functional in a canonical ensemble, where the finite volume pair correlation function and not the radial distribution function is the relevant stochastic quantity. To extend the uniqueness result to the radial distribution function, Henderson subsequently turns to the thermodynamical limit, ignoring the possibility that the strict inequality of the variational principle

*The research leading to this work has been funded by the Deutsche Forschungsgemeinschaft (DFG, German Research Foundation) - Projektnummer 233630050 - TRR 146.

†Institut für Mathematik, Johannes Gutenberg-Universität Mainz, 55099 Mainz, Germany (fafromme@uni-mainz.de)

${ }^{\ddagger}$ Institut für Mathematik, Johannes Gutenberg-Universität Mainz, 55099 Mainz, Germany (hanke@math.uni-mainz.de)

§Institut für Mathematik, Ludwig-Maximilians Universität München, Theresienstr. 39,80333 München, Germany (jansen@math.lmu.de). 
for finite volumes may turn into an equality when the volume tends to infinity. Accordingly, there is a gap in the argument provided in [10] - aside of the fact that no mention is made concerning the necessary requirements for the pair potential, e.g., its behavior for particle pairs with diminishing distances.

In this note we specify a suitably rich class of pair potentials, for which Henderson's approach can be turned into a rigorous proof by using a version of the Gibbs variational principle due to Georgii [5]. This class of potentials does include hard core potentials and the so-called Lennard-Jones type potentials, but is a strict subclass of all superstable potentials, cf., e.g., Ruelle [18] for this and further terminology. Strictly speaking, this means that our result does not answer the question whether the radial distribution function associated with, say, the classical Lennard-Jones potential can also occur for a much more exotic type of pair potential and the same values of temperature and density.

The thermodynamical limit may either be reached from a canonical or a grand canonical ensemble. We therefore also state a variant of Henderson's result which is more natural from the grand canonical perspective: It will be shown below that the pair potential is uniquely determined when given the temperature, the chemical potential, and the infinite volume pair correlation function; it is unknown whether in this second version of Henderson's statement the pair correlation function can be replaced by the radial distribution function in the isotropic case.

We mention that for the corresponding inverse problem on the lattice the uniqueness of the pair potential has already been settled by Griffiths and Ruelle [9]; see also Caglioti, Kuna, Lebowitz, and Speer [1].

The outline of this note is as follows: In Section 2 we review the rigorous mathematical setting of the thermodynamical limit of a grand canonical ensemble when the system is translation invariant and its potential energy is given by pairwise interactions only. Then we formulate in Section 3 the particular version of the Gibbs variational principle that is valid in this setting. Section 4 is devoted to the proof of the uniqueness results, and eventually we close with a few comments and open problems in Section 5.

2. The thermodynamical limit of the grand canonical ensemble. We start from a grand canonical ensemble of pointlike classical particles in a bounded box $\Lambda_{\ell}=[-\ell, \ell]^{d}$, with specified inverse temperature $\beta>0$ and chemical potential $\mu \in \mathbb{R}$. We restrict our attention to the case that the interaction of the particles is given by a pair potential $u: \mathbb{R}^{d} \rightarrow \mathbb{R} \cup\{+\infty\}$, which is an even function, i.e., $u(x)=u(-x)$, satisfying the following two assumptions:

1. There exists $r_{0}>0$ and a decreasing function $\varphi:\left(0, r_{0}\right] \rightarrow \mathbb{R}_{0}^{+}$with

$$
\int_{0}^{r_{0}} r^{d-1} \varphi(r) \mathrm{d} r=+\infty
$$

and

$$
u(x) \geq \varphi(|x|) \quad \text { for }|x| \leq r_{0} .
$$

2. There exists a decreasing function $\psi:\left[r_{0}, \infty\right) \rightarrow \mathbb{R}_{0}^{+}$with

$$
\int_{r_{0}}^{\infty} r^{d-1} \psi(r) \mathrm{d} r<\infty
$$

and

$$
|u(x)| \leq \psi(|x|) \quad \text { for }|x| \geq r_{0} .
$$


For this class $\mathscr{U}$ of potentials the associated configurational Hamiltonian of $m \in \mathbb{N}_{0}$ particles at positions $x_{i} \in \mathbb{R}^{d}, i=1, \ldots, m$, given by

$$
H_{u}\left(\mathbf{x}_{m}\right)=\sum_{1 \leq i<j \leq m} u\left(x_{i}-x_{j}\right), \quad \mathbf{x}_{m}=\left(x_{1}, \ldots, x_{m}\right),
$$

is stable (cf. Dobrushin [2]), i.e., for every $u \in \mathscr{U}$ there exists $B>0$ such that

$$
H_{u}\left(\mathbf{x}_{m}\right) \geq-B m
$$

independent of the number $m$ of particles. The statistical distribution of the particles of such a grand canonical ensemble is determined by the corresponding $m$-particle correlation functions

$$
\rho_{\Lambda_{\ell}}^{(m)}\left(\mathbf{x}_{m}\right)=\frac{e^{\beta \mu m}}{\Xi\left(\Lambda_{\ell}, \beta, \mu, u\right)} \sum_{N=0}^{\infty} \frac{e^{\beta \mu N}}{N !} \int_{\Lambda_{\ell}^{N}} e^{-\beta H_{u}\left(\mathbf{x}_{m}, \mathbf{y}_{N}\right)} \mathrm{d} \mathbf{y}_{N},
$$

where $\mathbf{x}_{m}=\left(x_{1}, \ldots, x_{m}\right) \in \Lambda_{\ell}^{m}, \mathbf{y}_{N}=\left(y_{1}, \ldots, y_{N}\right) \in \Lambda_{\ell}^{N}$, the integral $\int_{\Delta^{0}} c \mathrm{~d} \mathbf{x}_{0}$ with bounded domain $\Delta \subset \mathbb{R}^{d}$ is always taken to be equal to $c$, and the normalizing constant

$$
\Xi\left(\Lambda_{\ell}, \beta, \mu, u\right)=\sum_{N=0}^{\infty} \frac{e^{\beta \mu N}}{N !} \int_{\Lambda_{\ell}^{N}} e^{-\beta H_{u}\left(\mathbf{x}_{N}\right)} \mathrm{d} \mathbf{x}_{N}
$$

is the associated grand canonical partition function. In (2.2) $m$ varies in $\mathbb{N}_{0}$, with $\rho_{\Lambda_{\ell}}^{(0)}$ being set to one.

We assume that for some sequence $\left(\ell_{k}\right)_{k}$ going to infinity as $k \rightarrow \infty$, these correlation functions converge uniformly on compact subsets to translation invariant functions $\rho^{(m)}:\left(\mathbb{R}^{d}\right)^{m} \rightarrow \mathbb{R}_{0}^{+}, m \in \mathbb{N}_{0}$, defined on the entire space; in particular, this implies that $\rho^{(1)}$ is a constant. It is known that these limiting correlation functions satisfy a so-called Ruelle bound, i.e.,

$$
\sup _{\mathbf{x}_{m} \in\left(\mathbb{R}^{d}\right)^{m}} \rho^{(m)}\left(\mathbf{x}_{m}\right) \leq \xi^{m}, \quad m \in \mathbb{N}_{0},
$$

for some $\xi>0$, depending only on $\mu, \beta$, and $u$, and that they define a translation invariant probability measure $\mathbb{P}$ on the configuration space

$$
\Gamma=\left\{\gamma \subset \mathbb{R}^{d} \mid \Delta \subset \mathbb{R}^{d} \text { bounded } \Rightarrow \#(\gamma \cap \Delta)<\infty\right\},
$$

i.e., the set of all locally finite subsets of $\mathbb{R}^{d}$ representing the positions of the (at most countably many) individual particles in space, equipped with an appropriate $\sigma$-algebra, cf. Ruelle [19]. This means that if $m \in \mathbb{N}_{0}$ is fixed and an observable $F$ depends on all possible $m$-tuples of particles in a given configuration, i.e.,

$$
F(\gamma)=\sum_{\substack{x_{1}, \ldots, x_{m} \in \gamma \\ x_{i} \neq x_{j}}} f\left(\mathbf{x}_{m}\right)
$$

for some $f=f_{1}+f_{2}$ with $f_{1} \in L^{1}\left(\left(\mathbb{R}^{d}\right)^{m}\right)$ and $f_{2} \geq 0$, then

$$
\int_{\Gamma} F(\gamma) \mathrm{d} \mathbb{P}(\gamma)=\int_{\left(\mathbb{R}^{d}\right)^{m}} f\left(\mathbf{x}_{m}\right) \rho^{(m)}\left(\mathbf{x}_{m}\right) \mathrm{d} \mathbf{x}_{m}
$$


is the expected value of the corresponding observable. In particular, if $|\Delta|$ denotes the volume of any bounded domain $\Delta \subset \mathbb{R}^{d}$ then

$$
\rho(\mathbb{P})=\frac{1}{|\Delta|} \int_{\Gamma} \#(\gamma \cap \Delta) d \mathbb{P}(\gamma)=\rho^{(1)}
$$

is the limiting particle counting density.

According to [19], $\mathbb{P}$ is a translation invariant tempered $(\beta, \mu, u)$-Gibbs measure, denoted $\mathbb{P} \in \mathscr{G}(\beta, \mu, u)$. This means that $\mathbb{P}$ is supported by the set of tempered configurations (defined in [19]), and that for every $F \in L^{1}(\mathbb{P})$ and every bounded domain $\Delta \subset \mathbb{R}^{d}$ there holds

$$
\begin{aligned}
\int_{\Gamma} F(\gamma) \mathrm{d} \mathbb{P}(\gamma) \\
\quad=\sum_{N=0}^{\infty} \frac{z^{N}}{N !} \int_{\Delta^{N}}\left(\int_{\Gamma\left(\Delta^{c}\right)} F\left(\gamma^{\prime}\right) e^{-\beta W_{u}\left(\mathbf{x}_{N} ; \gamma\right)} \mathrm{d} \mathbb{P}(\gamma)\right) e^{-\beta H_{u}\left(\mathbf{x}_{N}\right)} \mathrm{d} \mathbf{x}_{N},
\end{aligned}
$$

where $\gamma^{\prime}=\gamma \cup\left\{x_{1}, \ldots, x_{N}\right\}, \Gamma\left(\Delta^{c}\right)=\left\{\gamma \in \Gamma: \gamma \subset \mathbb{R}^{d} \backslash \Delta\right\}$, and the interaction $W_{u}$ between particles at $x_{i}, i=1, \ldots, N$ and those of $\gamma \in \Gamma$ is defined as

$$
W_{u}\left(\mathbf{x}_{N} ; \gamma\right)=\sum_{i=1}^{N} \sum_{y \in \gamma} u\left(x_{i}-y\right),
$$

if the series converges absolutely, and as $+\infty$ otherwise.

Given the limiting correlation functions one can define Janossy densities $j_{\Lambda_{\ell}}^{(m)}$ : $\Lambda_{\ell}^{m} \rightarrow \mathbb{R}$ for every $m \in \mathbb{N}_{0}$ and $\ell>0$ via

$$
j_{\Lambda_{\ell}}^{(m)}\left(\mathbf{x}_{m}\right)=\sum_{k=0}^{\infty} \frac{(-1)^{k}}{k !} \int_{\Lambda_{\ell}^{k}} \rho^{(m+k)}\left(\mathbf{x}_{m}, \mathbf{y}_{k}\right) \mathrm{d} \mathbf{y}_{k} .
$$

These Janossy densities provide the induced probability density on $\Lambda_{\ell}$, for which

$$
\int_{\Gamma} F(\gamma) \mathrm{d} \mathbb{P}(\gamma)=\sum_{m=0}^{\infty} \frac{1}{m !} \int_{\Lambda_{\ell}^{m}} f_{m}\left(\mathbf{x}_{m}\right) j_{\Lambda_{\ell}}^{(m)}\left(\mathbf{x}_{m}\right) \mathrm{d} \mathbf{x}_{m}
$$

for every $F \in L^{1}(\mathbb{P})$, which satisfies $F(\gamma)=F\left(\gamma \cap \Lambda_{\ell}\right)$, and which is given by functions $f_{m}: \Lambda^{m} \rightarrow \mathbb{R}, m \in \mathbb{N}_{0}$, such that $F\left(\gamma_{m}\right)=f_{m}\left(\mathbf{x}_{m}\right)$, when $\gamma_{m}=\left\{x_{1}, \ldots, x_{m}\right\} \subset \Lambda$. Such observables $F$ are thus called local observables.

Varying $\beta>0, \mu \in \mathbb{R}$, and $u \in \mathscr{U}$, we denote by

$$
\mathscr{G}=\bigcup_{\beta, \mu, u} \mathscr{G}(\beta, \mu, u)
$$

the union of all translation invariant tempered Gibbs measures, some of which may not be obtained as limits of finite-volume Gibbs measures with empty boundary conditions (cf., e.g., Georgii [4]). We mention for later use that for almost every $x \in \mathbb{R}^{d}$ and $\mathbb{P}$ almost surely for every $\mathbb{P} \in \mathscr{G}$ the interaction defined in (2.6) is finite, and there holds

$$
\lim _{\ell \rightarrow \infty} W_{u}\left(x ; \gamma \cap \Lambda_{\ell}\right)=W_{u}(x ; \gamma),
$$

see [12, Sect. 5]. We mention further that there exists some $\mu_{0} \in \mathbb{R}$ depending on $\beta$ and $u \in \mathscr{U}$, such that for $\mu<\mu_{0}$ - the so-called gas phase - the set $\mathscr{G}(\beta, \mu, u)$ consists of a single Gibbs measure $\mathbb{P}$ only, cf. [19], and that in this case the correlation functions $\rho_{\Lambda_{\ell}}^{(m)}$ converge to $\rho^{(m)}$ as $\ell \rightarrow \infty$ for every $m \in \mathbb{N}_{0}$, cf. [18]. 
3. The Gibbs variational principle. The Gibbs variational principle goes back to Gibbs' work (cf. [8, p. 131]) and appears in different variants in statistical mechanics and stochastic analysis; we refer, e.g., to the books by Ruelle [18], Gallavotti [3], and Georgii [6] for rigorous mathematical treatments of this variational principle. Here we apply a particular version established by Georgii and Zessin in the series of papers $[7,4,5]$.

For pair potentials $u \in \mathscr{U}$ and Gibbs measures $\mathbb{P} \in \mathscr{G}$, we introduce the specific energy

$$
E(u, \mathbb{P})=\lim _{\ell \rightarrow \infty} \frac{1}{\left|\Lambda_{\ell}\right|} \int_{\Gamma} \frac{1}{2} \sum_{\substack{x, y \in \gamma \cap \Lambda_{\ell} \\ x \neq y}} u(x-y) \mathrm{d} \mathbb{P}(\gamma)
$$

and the specific (relative) entropy

$$
S(\mathbb{P})=\lim _{\ell \rightarrow \infty} \frac{1}{\left|\Lambda_{\ell}\right|} \sum_{m=0}^{\infty} \frac{1}{m !} \int_{\Lambda_{\ell}^{m}} j_{\Lambda_{\ell}}^{(m)}\left(\mathbf{x}_{m}\right) \log \left(j_{\Lambda_{\ell}}^{(m)}\left(\mathbf{x}_{m}\right)\right) \mathrm{d} \mathbf{x}_{m},
$$

where both limits are known to exist in $\mathbb{R} \cup\{+\infty\}$ : concerning the specific energy see Proposition 3.1 below; concerning the specific entropy we refer to Robinson and Ruelle [17] - in fact, using (2.7) and (2.3) it is not too difficult to see that $S(\mathbb{P})$ is finite for every $\mathbb{P} \in \mathscr{G}$. The relative entropy differs from the standard entropy by a sign, to simplify language we call $S(P)$ nevertheless the entropy. We take similar liberties with the sign of the (specific) grand potential

$$
\Omega_{\beta, \mu}(u, \mathbb{P})=\mu \rho(\mathbb{P})-E(u, \mathbb{P})-\frac{1}{\beta} S(\mathbb{P}),
$$

for which the following variational principle holds true ([5, Theorem 3.4]).

Theorem A. For fixed $\mu \in \mathbb{R}, \beta>0$, and $u \in \mathscr{U}$ the grand potential $\Omega_{\beta, \mu}(u, \cdot)$ has values in $\mathbb{R} \cup\{-\infty\}$. Its maximal value $p$ on $\mathscr{G}$ is attained for every $\mathbb{P} \in \mathscr{G}(\beta, \mu, u)$, and there holds

$$
\Omega_{\beta, \mu}(u, \mathbb{P})<p
$$

for every other $\mathbb{P} \in \mathscr{G}$. Here,

$$
p=\lim _{\ell \rightarrow \infty} \frac{1}{\beta\left|\Lambda_{\ell}\right|} \log \Xi\left(\Lambda_{\ell}, \beta, \mu, u\right)
$$

is the pressure in the thermodynamical limit.

For the proof of the Henderson result we will also need the following identity, for which we include a self-contained proof for the ease of the reader.

Proposition 3.1. For every $u \in \mathscr{U}$ and every $\mathbb{P} \in \mathscr{G}$ the limit in (3.1) belongs to $\mathbb{R} \cup\{+\infty\}$, and is given by

$$
E(u, \mathbb{P})=\frac{1}{2} \int_{\mathbb{R}^{d}} u(x) \rho^{(2)}(x, 0) \mathrm{d} x,
$$

where $\rho^{(2)}$ is the pair correlation function associated with $\mathbb{P}$.

Proof. Let $\rho^{(2)}$ be the translation invariant pair correlation function associated with $\mathbb{P}$. Then we can apply (2.4) with $m=2$ and

$$
f(x, y)= \begin{cases}u(x-y), & x, y \in \Lambda_{\ell} \\ 0, & \text { else }\end{cases}
$$


to rewrite

$$
\begin{aligned}
& \int_{\Gamma} \sum_{\substack{x, y \in \gamma \cap \Lambda_{\ell} \\
x \neq y}} u(x-y) \mathrm{d} \mathbb{P}(\gamma)=\int_{\Lambda_{\ell}^{2}} u(x-y) \rho^{(2)}(x, y) \mathrm{d}(x, y) \\
& =\int_{\Lambda_{\ell}}\left(\int_{\Delta_{x, \ell}} u(x-y) \rho^{(2)}(x, y) \mathrm{d} y+\int_{\Lambda_{\ell} \backslash \Delta_{x, \ell}} u(x-y) \rho^{(2)}(x, y) \mathrm{d} y\right) \mathrm{d} x,
\end{aligned}
$$

where the set $\Delta_{x, \ell}=\left\{y \in \Lambda_{\ell} \mid u(x-y) \geq 1\right\}$ is bounded, and $u(x-\cdot)$ is absolutely integrable over $\mathbb{R}^{d} \backslash \Delta_{x, \ell}$ because of (2.1). Therefore, and since $\rho^{(2)}$ is bounded, compare (2.3), the second inner integral in (3.4) is uniformly bounded, independent of $\ell$ and $x \in \Lambda_{\ell}$. The integrand of the first inner integral is nonnegative. In case this integral diverges for some $\ell \in \mathbb{N}$ and some $x \in \Lambda_{\ell}$ then the total right-hand side of (3.4) equals $+\infty$, and this remains true for all larger values of $\ell$, i.e., $E(u, \mathbb{P})=+\infty$. The same argument applied to the right-hand side of (3.3) shows that equality holds in (3.3) in this case, because $\rho^{(2)}$ is translation invariant.

On the other hand, if the inner integral over $\Delta_{x, \ell}$ in (3.4) is finite for every $\ell \in \mathbb{N}$ and every $x \in \Lambda_{\ell}$, then the same argument as before, together with the translation invariance of $\rho^{(2)}$ shows that

$$
\int_{\mathbb{R}^{d}} u(x-y) \rho^{(2)}(x, y) \mathrm{d} y=\int_{\mathbb{R}^{d}} u(y) \rho^{(2)}(y, 0) \mathrm{d} y
$$

is absolutely convergent. Now we assume that $\ell$ is greater than the parameter $r_{0}$ which occurs in (2.1). Then we choose some $r$ between $r_{0}$ and $\ell$, and we split the domain $\Lambda_{\ell}^{2}$ of integration into

$$
\Lambda_{\ell}^{2}=\Delta_{1} \cup \Delta_{2} \cup \Delta_{3}
$$

where

$$
\begin{aligned}
& \Delta_{1}=\left\{(x, y) \in \Lambda_{\ell}^{2}: x \in \Lambda_{\ell-r},|y-x| \leq r\right\}, \\
& \Delta_{2}=\left\{(x, y) \in \Lambda_{\ell}^{2}: x \in \Lambda_{\ell} \backslash \Lambda_{\ell-r},|y-x| \leq r\right\} \\
& \Delta_{3}=\left\{(x, y) \in \Lambda_{\ell}^{2}:|x-y|>r\right\} .
\end{aligned}
$$

Under these assumptions on $r$ and $\ell$ it follows from the fact that (3.5) is absolutely convergent that

$$
\begin{aligned}
& \left|\int_{\Delta_{2}} u(x-y) \rho^{(2)}(x, y) \mathrm{d}(x, y)\right| \leq \int_{\Lambda_{\ell} \backslash \Lambda_{\ell-r}} \int_{|y-x| \leq r}|u(x-y)| \rho^{(2)}(x, y) \mathrm{d} y \mathrm{~d} x \\
& \quad \leq\left(\left|\Lambda_{\ell}\right|-\left|\Lambda_{\ell-r}\right|\right) \int_{\mathbb{R}^{3}}|u(y)| \rho^{(2)}(y, 0) \mathrm{d} y
\end{aligned}
$$

and

$$
\begin{aligned}
& \left|\int_{\Delta_{3}} u(x-y) \rho^{(2)}(x, y) \mathrm{d}(x, y)\right| \leq \int_{\Delta_{3}}|u(x-y)| \rho^{(2)}(x, y) \mathrm{d}(x, y) \\
& \quad \leq \int_{\Lambda_{\ell}} \int_{|y-x|>r}|u(x-y)| \rho^{(2)}(x, y) \mathrm{d} y \mathrm{~d} x \leq\left|\Lambda_{\ell}\right| \int_{|y|>r}|u(y)| \rho^{(2)}(y, 0) \mathrm{d} y .
\end{aligned}
$$


Since (3.5) converges absolutely we can thus choose $r=r(\varepsilon)$ sufficiently large to make sure that

$$
\limsup _{\ell \rightarrow \infty}\left|\frac{1}{\left|\Lambda_{\ell}\right|} \int_{\Delta_{2} \cup \Delta_{3}} u(x-y) \rho^{(2)}(x, y) \mathrm{d}(x, y)\right| \leq \varepsilon
$$

for any given positive number $\varepsilon$. On the other hand, using the translation invariance again, we have

$$
\begin{aligned}
\int_{\Delta_{1}} u & (x-y) \rho^{(2)}(x, y) \mathrm{d}(x, y)=\int_{\Lambda_{\ell-r}} \int_{|y-x| \leq r} u(x-y) \rho^{(2)}(x, y) \mathrm{d} y \mathrm{~d} x \\
& =\left|\Lambda_{\ell-r}\right| \int_{|y| \leq r} u(y) \rho^{(2)}(y, 0) \mathrm{d} y
\end{aligned}
$$

and hence,

$$
\begin{aligned}
& \limsup _{\ell \rightarrow \infty}\left|\frac{1}{\left|\Lambda_{\ell}\right|} \int_{\Delta_{1}} u(x-y) \rho^{(2)}(x, y) \mathrm{d}(x, y)-\int_{\mathbb{R}^{d}} u(y) \rho^{(2)}(y, 0) \mathrm{d} y\right| \\
& \quad \leq \int_{|y|>r}|u(y)| \rho^{(2)}(y, 0) \mathrm{d} y .
\end{aligned}
$$

Combining (3.7) for $r=r(\varepsilon)$ with (3.6), the assertion (3.3) follows by letting $\varepsilon \rightarrow 0$.

口

We mention that the Kirkwood-Salsburg equations (cf., e.g., [19]) can be used to argue that the integrand of (3.3) is bounded near the origin when $\mathbb{P}$ is a $(\beta, \mu, u)$-Gibbs measure, so that for "matching" $u$ and $\mathbb{P}$ the integral is absolutely convergent and finite by virtue of (2.1) and (2.3).

4. Uniqueness results of Henderson type in the thermodynamical limit. We now formulate Henderson's theorem in the spirit of his original paper [10], and provide a rigorous proof, based on arguments borrowed from [10] and from the proof of [6, Thm. 2.34].

TheOREm 4.1. Let $u, v \in \mathscr{U}, \beta>0$, and $\mu, \mu^{\prime} \in \mathbb{R}$ be given, and assume that $\mathbb{P}_{u} \in \mathscr{G}(\beta, \mu, u)$ and $\mathbb{P}_{v} \in \mathscr{G}\left(\beta, \mu^{\prime}, v\right)$ admit the same density $\rho^{(1)}$ and the same pair correlation function $\rho^{(2)}$. Then $\mu=\mu^{\prime}$ and $u=v$ almost everywhere.

Proof. By Theorem A we have

$$
\Omega_{\beta, \mu}\left(u, \mathbb{P}_{v}\right) \leq \Omega_{\beta, \mu}\left(u, \mathbb{P}_{u}\right) \quad \text { and } \quad \Omega_{\beta, \mu^{\prime}}\left(v, \mathbb{P}_{u}\right) \leq \Omega_{\beta, \mu^{\prime}}\left(v, \mathbb{P}_{v}\right)
$$

Since $\Omega_{\beta, \mu}\left(u, \mathbb{P}_{u}\right)$ and $\Omega_{\beta, \mu^{\prime}}\left(v, \mathbb{P}_{v}\right)$ are finite we may write these inequalities as

$$
\Omega_{\beta, \mu}\left(u, \mathbb{P}_{v}\right)-\Omega_{\beta, \mu}\left(u, \mathbb{P}_{u}\right) \leq 0 \quad \text { and } \quad \Omega_{\beta, \mu^{\prime}}\left(v, \mathbb{P}_{u}\right)-\Omega_{\beta, \mu^{\prime}}\left(v, \mathbb{P}_{v}\right) \leq 0,
$$

and adding them we get

$$
\Omega_{\beta, \mu}\left(u, \mathbb{P}_{v}\right)-\Omega_{\beta, \mu}\left(u, \mathbb{P}_{u}\right)+\Omega_{\beta, \mu^{\prime}}\left(v, \mathbb{P}_{u}\right)-\Omega_{\beta, \mu^{\prime}}\left(v, \mathbb{P}_{v}\right) \leq 0 .
$$


Recalling the definition (3.2) of the grand potential we have

$$
\begin{aligned}
\Omega_{\beta, \mu}\left(u, \mathbb{P}_{v}\right)-\Omega_{\beta, \mu}\left(u, \mathbb{P}_{u}\right)+\Omega_{\beta, \mu^{\prime}}\left(v, \mathbb{P}_{u}\right)-\Omega_{\beta, \mu^{\prime}}\left(v, \mathbb{P}_{v}\right) \\
=\mu \rho\left(\mathbb{P}_{v}\right)-E\left(u, \mathbb{P}_{v}\right)-\frac{1}{\beta} S\left(\mathbb{P}_{v}\right)-\mu \rho\left(\mathbb{P}_{u}\right)+E\left(u, \mathbb{P}_{u}\right)+\frac{1}{\beta} S\left(\mathbb{P}_{u}\right) \\
\quad+\mu^{\prime} \rho\left(\mathbb{P}_{u}\right)-E\left(v, \mathbb{P}_{u}\right)-\frac{1}{\beta} S\left(\mathbb{P}_{u}\right)-\mu^{\prime} \rho\left(\mathbb{P}_{v}\right)+E\left(v, \mathbb{P}_{v}\right)+\frac{1}{\beta} S\left(\mathbb{P}_{v}\right) \\
=-E\left(u, \mathbb{P}_{v}\right)+E\left(u, \mathbb{P}_{u}\right)-E\left(v, \mathbb{P}_{u}\right)+E\left(v, \mathbb{P}_{v}\right),
\end{aligned}
$$

because $\rho\left(\mathbb{P}_{u}\right)=\rho\left(\mathbb{P}_{v}\right)=\rho^{(1)}$ by assumption. Furthermore, by virtue of Proposition 3.1 and the fact that the pair correlation functions of $\mathbb{P}_{u}$ and $\mathbb{P}_{v}$ coincide, there holds

$$
E\left(u, \mathbb{P}_{u}\right)=\frac{1}{2} \int_{\mathbb{R}^{d}} u(x) \rho^{(2)}(x, 0) \mathrm{d} x=E\left(u, \mathbb{P}_{v}\right)
$$

and

$$
E\left(v, \mathbb{P}_{u}\right)=\frac{1}{2} \int_{\mathbb{R}^{d}} v(x) \rho^{(2)}(x, 0) \mathrm{d} x=E\left(v, \mathbb{P}_{v}\right) .
$$

Inserting this into equation (4.3) we conclude that

$$
\Omega_{\beta, \mu}\left(u, \mathbb{P}_{v}\right)-\Omega_{\beta, \mu}\left(u, \mathbb{P}_{u}\right)+\Omega_{\beta, \mu^{\prime}}\left(v, \mathbb{P}_{u}\right)-\Omega_{\beta, \mu^{\prime}}\left(v, \mathbb{P}_{v}\right)=0 .
$$

Accordingly, equality holds in (4.2), and thus necessarily in both statements of equation (4.1). By the Gibbs variational principle (Theorem A) this implies that $\mathbb{P}_{v} \in \mathscr{G}(\beta, \mu, u)$ and $\mathbb{P}_{u} \in \mathscr{G}\left(\beta, \mu^{\prime}, v\right)$.

It therefore follows from (2.5) that

$$
\begin{aligned}
\int_{\Gamma} F(\gamma) \mathrm{d} \mathbb{P}_{u}(\gamma) & =\sum_{N=0}^{\infty} \frac{1}{N !} \int_{\Delta^{N}}\left(\int_{\Gamma\left(\Delta^{c}\right)} F\left(\gamma^{\prime}\right) e^{-\beta W_{u}\left(\mathbf{x}_{N} ; \gamma\right)} \mathrm{d} \mathbb{P}_{u}(\gamma)\right) e^{N \beta \mu-\beta H_{u}\left(\mathbf{x}_{N}\right)} \mathrm{d} \mathbf{x}_{N} \\
& =\sum_{N=0}^{\infty} \frac{1}{N !} \int_{\Delta^{N}}\left(\int_{\Gamma\left(\Delta^{c}\right)} F\left(\gamma^{\prime}\right) e^{-\beta W_{v}\left(\mathbf{x}_{N} ; \gamma\right)} \mathrm{d} \mathbb{P}_{u}(\gamma)\right) e^{N \beta \mu^{\prime}-\beta H_{v}\left(\mathbf{x}_{N}\right)} \mathrm{d} \mathbf{x}_{N}
\end{aligned}
$$

for every $F \in L^{1}\left(\mathbb{P}_{u}\right)$ and every bounded domain $\Delta \subset \mathbb{R}^{d}$. Therefore

$$
H_{u}\left(\mathbf{x}_{N}\right)+W_{u}\left(\mathbf{x}_{N} ; \gamma\right)-N \mu=H_{v}\left(\mathbf{x}_{N}\right)+W_{v}\left(\mathbf{x}_{N} ; \gamma\right)-N \mu^{\prime}
$$

for every $N \in \mathbb{N}$, almost every $\mathbf{x}_{N} \in \Delta^{N}$ and $\mathbb{P}_{u}$ almost surely for $\gamma \in \Gamma\left(\Delta^{c}\right)$. For $N=1$ this means that

$$
W_{u}(x ; \gamma)-\mu=W_{v}(x ; \gamma)-\mu^{\prime}
$$

for almost every $x \in \Delta$ and $\mathbb{P}_{u}$ and $\mathbb{P}_{v}$ almost surely for $\gamma \in \Gamma\left(\Delta^{c}\right)$. Using the additivity of $W_{u}$ and $W_{v}$ in the first argument we thus conclude from the case $N=2$ of (4.4) that

$$
u(x-y)=v(x-y)
$$

for almost every $x, y \in \Delta$, and since $\Delta$ was arbitrarily chosen, we have $u=v$ almost everywhere. 
Inserting (4.6) into (2.6) it follows that

$$
W_{u}\left(x ; \gamma_{0}\right)=W_{v}\left(x ; \gamma_{0}\right)
$$

for almost every $x \in \Delta$ and almost every finite subset $\gamma_{0} \subset \Delta^{c} \cap \Lambda_{\ell}$. Together with (2.8) this implies that for every $\mathbb{P} \in \mathscr{G}$ there holds

$$
W_{u}\left(x ; \gamma \cap \Lambda_{\ell}\right)=W_{v}\left(x ; \gamma \cap \Lambda_{\ell}\right)
$$

$\mathbb{P}$ almost surely for $\gamma \in \Gamma\left(\Delta^{c}\right)$. By virtue of (2.9) and (4.7) we therefore have

$$
W_{u}(x ; \gamma)=W_{v}(x ; \gamma)
$$

for almost every $x \in \Delta$ and $\mathbb{P}_{u}$ almost surely for $\gamma \in \Gamma\left(\Delta^{c}\right)$, and hence we conclude from (4.5) that $\mu=\mu^{\prime}$, which remained to be shown.

Henderson stipulated the assumptions of Theorem 4.1 from the canonical ensemble point of view. Concerning the grand canonical perspective an analogous uniqueness result is as follows.

TheOREM 4.2. Let $u, v \in \mathscr{U}, \beta>0$, and $\mu \in \mathbb{R}$ be given, and assume that $\mathbb{P}_{u} \in \mathscr{G}(\beta, \mu, u)$ and $\mathbb{P}_{v} \in \mathscr{G}(\beta, \mu, v)$ admit the same pair correlation function $\rho^{(2)}$. Then $u=v$ almost everywhere.

The proof is the same as for Theorem 4.1: This time (4.3) holds true because the chemical potentials are the same. We mention, however, that we do not know whether the counting densities of the two Gibbs measures are necessarily the same, unless it is assumed that the corresponding $(\beta, \mu, u)$-Gibbs measure is uniquely determined as it is, e.g., in the gas phase.

5. Concluding Remarks. We emphasize that for our results we do not stipulate that the system is in the gas phase, nor that the $\operatorname{set} \mathscr{G}(\beta, \mu, u)$ consists of a single Gibbs measure only.

In case it is known that $u$ is also radially symmetric, i.e., if the interaction of two particles only depends on their distance, then one can show - using the MarkovKakutani fixed point theorem as in the proof of [18, Theorem 5.8], compare Kuna [14] - that there exists at least one rotation and translation invariant Gibbs measure $\mathbb{P}_{u} \in$ $\mathscr{G}(\beta, \mu, u)$, which can be used to define a radial distribution function $g: \mathbb{R}^{+} \rightarrow \mathbb{R}_{0}^{+}$ given by

$$
g(r)=\frac{\rho^{(2)}\left(x_{1}, x_{2}\right)}{\left(\rho^{(1)}\right)^{2}}, \quad r=\left|x_{1}-x_{2}\right|,
$$

provided that the density is nonzero. Assuming further that $\mathbb{P}_{v} \in \mathscr{G}(\beta, \mu, v)$ is also rotation invariant, then one obviously can impose in Theorem 4.1 - as did Henderson - that the radial distribution functions and the densities are the same for these two Gibbs measures, and the statement of the theorem remains valid. We do not know whether in the formulation of Theorem $4.2 \rho^{(2)}$ can also be replaced by the radial distribution function in the isotropic case.

Finally we remark that the representation (3.3) of the specific energy is not essential for the uniqueness argument. By its definition (3.1), the specific energy $E(u, \mathbb{P})$ is the limit in $\mathbb{R} \cup\{+\infty\}$ of the expression given in (3.4) normalized by two times the volume of $\Lambda_{\ell}$, and hence, its value only depends on $u$ and on the pair correlation function $\rho^{(2)}$ associated with $\mathbb{P}$. This suffices to conclude that the expression (4.3) sums up to zero. 
Having settled the uniqueness problem the natural follow-up question concerns the existence of solutions of the inverse Henderson problem, i.e., what are necessary and sufficient conditions on a given triplet $\beta, \rho>0, \mu \in \mathbb{R}$, and a nonnegative translation invariant function $\rho^{(2)}: \mathbb{R}^{2} \rightarrow \mathbb{R}$, such that there exists a pair potential $u \in \mathscr{U}$ for which $\rho$ is the density and $\rho^{(2)}$ is the pair correlation function of a Gibbs measure $\mathbb{P} \in \mathscr{G}(\beta, \mu, u)$. Partial results for this problem have been contributed, e.g., by Caglioti, Kuna, Lebowitz, and Speer [1], Kuna, Lebowitz and Speer [15], and Koralov [13]. The general existence problem is widely open, though.

\section{REFERENCES}

[1] E. Caglioti, T. Kuna, J. Lebowitz, and E. Speer, Point processes with specified low order correlations, Markov Process. Related Fields, 12 (2006), pp. 257-272.

[2] R. Dobrushin, Investigation of conditions for the asymptotic existence of the configuration integral of Gibbs' distribution, Theor. Probability Appl., 9 (1964), pp. 566-581.

[3] G. Gallavotti, Statistical Mechanics: A Short Treatise, Springer, Berlin, 1999.

[4] H.-O. GEORGII, Large deviations and the equivalence of ensembles for Gibbsian particle systems with superstable interaction, Probab. Theory Related Fields, 99 (1994), pp. 171-195.

[5] - The equivalence of ensembles for classical systems of particles, J. Stat. Phys., 80 (1995), pp. 1341-1378.

[6] - Gibbs measures and phase transitions, De Gruyter, Berlin, 2. ed., 2011.

[7] H.-O. Georgin And H. Zessin, Large deviations and the maximum entropy principle for marked point random fields, Probab. Theory Related Fields, 96 (1993), pp. 177-204.

[8] J. GibBs, Elementary Principles in Statistical Mechanics, Scribner's Sons, New York, 1902.

[9] R. GRiffiths And D. Ruelle, Strict convexity ("continuity") of the pressure in lattice systems, Comm. Math. Phys., 23 (1971), pp. 169-175.

[10] R. Henderson, A uniqueness theorem for fluid pair correlation functions, Phys. Lett. A, 49 (1974), pp. 197-198.

[11] P. Hohenberg And W. Kohn, Inhomogeneous electron gas, Phys. Rev., 136 (1964), pp. B 864B 871.

[12] Y. G. Kondratiev and T. Kuna, Correlation functionals for Gibbs measures and Ruelle bounds, Methods Funct. Anal. Topology, 9 (2003), pp. 9-58.

[13] L. Koralov, An inverse problem for Gibbs fields with hard core potential, J. Math. Phys., 48 (2007). 053301.

[14] T. KunA, Studies in configuration space analysis and applications, $\mathrm{PhD}$ thesis, Rheinische Friedrich-Wilhelms-Universität, Bonn, 1999.

[15] T. Kuna, J. Lebowitz, and E. Speer, Realizability of point processes, J. Stat. Phys., 129 (2007), pp. 417-439.

[16] N. Mermin, Thermal properties of the inhomogeneous electron gas, Phys. Rev., 137 (1965), pp. A $1471-\mathrm{A} 1473$.

[17] D. W. Robinson and D. Ruelle, Mean entropy of states in classical statistical mechanics, Comm. Math. Phys., 5 (1967), pp. 288-300.

[18] D. Ruelle, Statistical Mechanics: Rigorous Results, W.A. Benjamin Publ., New York, 1969.

[19] - Superstable interactions in classical statistical mechanics, Comm. Math. Phys., 18 (1970), pp. 127-159. 